\title{
COMPLEX INTERPOLATING POLYNOMIALS
}

\author{
A. K. VARMA \\ (Communicated by Richard R. Goldberg) \\ Dedicated to Professors A. Zygmund and G. G. Lorentz
}

\begin{abstract}
Let $I_{n, m}(f, z)$ be the unique interpolatory polynomial of degree $\leq 2 n-1$ satisfying the conditions given by (1.1) where the $z_{k n}$ 's are the zeros of the polynomial $z^{n}-1$. The object of this paper is to consider the rate of convergence of $I_{n, m}(f, z)$ to $f(z)$ in the $L_{p}$ norm where $f \in C[|z| \leq 1]$. This problem was initially raised by $\mathrm{P}$. Turán in the case $p=2$ and in this case the solution was obtained by J. Szabados and A. K. Varma in [7].
\end{abstract}

Let $f(z)$ be an analytic function in the open unit circle $|z|<1$ and continuous in $|z| \leq 1$. (This class of functions will be denoted by $C[|z| \leq 1]$.) The main results of this paper can be described as follows.

THEOREM 1. Let $f(z) \in C[|z| \leq 1]$, and let $I_{n, m}(f, z)$ be the unique interpolatory polynomial of degree $\leq 2 n-1$ satisfying the conditions

$$
I_{n, m}\left(f, z_{k n}\right)=f\left(z_{k n}\right), \quad I_{n, m}^{(m)}\left(f, z_{k n}\right)=0, \quad k=1,2, \ldots, n,
$$

where

$$
z_{k n}=e^{2 \pi i k / n}, \quad k=1,2, \ldots, n, \quad \theta_{k n}=2 \pi k / n,
$$

are the zeros of the polynomial $z^{n}-1=0$. Then for any fixed $0<p<\infty$ we have

$$
\left(\frac{1}{2 \pi} \int_{C:|z|=1}\left|f(z)-I_{n, m}(f, z)\right|^{p}|d z|\right)^{1 / p} \leq c w_{m}\left(f, \frac{1}{n}\right)
$$

where $c$ is a positive constant depending only on $p$. Here $w_{m}(f, \delta)$ is the generalized modulus of continuity of $f\left(e^{i \theta}\right), 0 \leq \theta \leq 2 \pi$.

REMARK 1. In the case of $p=2$, the above theorem reduces to the one obtained earlier [7] in a joint work with J. Szabados (with $w(f, 1 / n)$ on the right side of the inequality (1.3)). The method of proof is very different than the one used earlier. Moreover, as mentioned in [7], the solution of Turán's open problem XLVI [8] is indeed a special case of Theorem 1.

We shall also prove the following.

THEOREM 2. Let $f(z) \in C[|z| \leq 1]$, and let $R_{n, m}(f, z)$ be the unique interpolatory polynomial of degree $\leq 2 n-1$ satisfying the conditions

$$
R_{n, m}\left(f, z_{k n}\right)=f\left(z_{k n}\right), \quad R_{n, m}^{(m)}\left(f, z_{k n}\right)=\beta_{k n} \quad(k=1,2, \ldots, n) .
$$

Received by the editors February 24, 1987.

1980 Mathematics Subject Classification (1985 Revision). Primary 41A05; Secondary 30E05, $30 \mathrm{E} 10$. 
Then we have

$$
\lim _{n \rightarrow \infty}\left(\frac{1}{2 \pi} \int_{C:|z|=1}\left|f(z)-R_{n, m}(f, z)\right|^{p}|d z|\right)^{1 / p}=0, \quad 0<p<\infty
$$

provided

$$
\beta_{k n}=o\left(n^{m}\right), \quad k=1,2, \ldots, n .
$$

REMARK 2. From earlier results of Riemenschneider and A. Sharma [4, Theorem 4.1], it follows that if $\beta_{k n}=o\left(n^{m} / \log n\right), f(z) \in C[|z| \leq 1]$ together with some Dini Lipschitz condition then $R_{n}(f, z)$ converges uniformly to $f(z)$ on $|z| \leq 1$. Thus in the case of $L_{p}$ convergence of $R_{n}(f, z)$ to $f(z)$ we have gained more freedom of $\beta_{k n}$. We can also drop the Dini Lipschitz condition of $f(z)\left(z=e^{i \theta}\right)$.

2. Preliminaries. It is known that $[3,4]$

$$
I_{n, m}(f, z)=\sum_{k=1}^{n} f\left(z_{k n}\right) A_{k, n, m}(z)
$$

and

$$
R_{n, m}(f, z)=\sum_{k=1}^{n} f\left(z_{k n}\right) A_{k, n, m}(z)+\sum_{k=1}^{n} \beta_{k n m} B_{k, n, m}(z)
$$

where

$$
\begin{aligned}
& A_{k, n, m}(z)=l_{k n}(z)-\frac{1}{n} \sum_{\nu=0}^{n-1} \lambda_{\nu, n, m} z_{k n}^{n-\nu} z^{\nu}\left(z^{n}-1\right) \\
& B_{k, n, m}(z)=\frac{\left(z^{n}-1\right)}{n} \sum_{\nu=0}^{n-1} z_{k n}^{m-\nu} \mu_{\nu, n, m} z^{\nu}
\end{aligned}
$$

Here

$$
\begin{aligned}
& l_{k n}(z)=\frac{z_{k n}}{n} \frac{z^{n}-1}{z-z_{k n}}=\frac{1}{n} \sum_{\nu=0}^{n-1}\left(\frac{z}{z_{k n}}\right)^{\nu} \\
& \lambda_{\nu, n, m}=\frac{(\nu)_{m}}{(\nu+n)_{m}-(\nu)_{m}}, \quad \mu_{\nu, n, m}=\frac{1}{(\nu+n)_{m}-(\nu)_{m}} \\
& (\nu)_{m}=\nu(\nu-1) \cdots(\nu-m+1) .
\end{aligned}
$$

We also note from (2.6) and (2.7)

$$
\left|\lambda_{j, n, m}\right| \leq c_{1}, \quad\left|\mu_{j, n, m}\right| \leq c_{2} / n^{m}
$$$$
\left|\lambda_{n-1, n, m}-\lambda_{n, n, m}\right| \leq c_{3} / n, \quad\left|\mu_{n-1, n, m}-\mu_{n, n, m}\right| \leq c_{4} / n^{m+1} \text {, }
$$$$
\left|\lambda_{j-1, n, m}-2 \lambda_{j, n, m}+\lambda_{j+1, n, m}\right| \leq c_{5} / n^{2},
$$$$
\left|\mu_{j-1, n, m}-2 \mu_{j, n, m}+\mu_{j+1, n, m}\right| \leq c_{6} / n^{m+2},
$$

where $c_{1}, c_{2}, \ldots$ are all absolute constants.

Let us denote by

$$
t_{1, k}(\theta)=1, \quad t_{\nu, k}(\theta)=1+\frac{2}{\nu} \sum_{j=1}^{\nu-1}(\nu-j) \cos j\left(\theta-\theta_{k n}\right), \quad 1 \leq \nu \leq n
$$


the well-known Féjer kernel, also $\tilde{t}_{\nu, k}(\theta)$ is the trigonometric polynomial conjugate to $t_{\nu, k}(\theta)$. We set

$$
p_{1, k}(z)=1, \quad p_{\nu, k}(z)=1+\frac{2}{\nu} \sum_{j=1}^{\nu-1}(\nu-j)\left(\frac{z}{z_{k n}}\right)^{j}, \quad \nu=2,3, \ldots
$$

Then a simple computation shows that

$$
\begin{aligned}
& p_{\nu, k}(z)=t_{\nu, k}(\theta)+i \tilde{t}_{\nu, k}(\theta), \quad z=e^{i \theta} \\
& 2\left(z / z_{k n}\right)^{\nu}=(\nu+1) p_{\nu+1, k}(z)-2 \nu p_{\nu, k}(z)+(\nu-1) p_{\nu-1, k}(z)
\end{aligned}
$$

and

$$
n p_{n, k}(z)=(n-1) p_{n-1, k}(z)-1+2 n l_{k n}(z) .
$$

3. Some lemmas. The following lemmas are needed for the proof of our theorems.

LEMMA 3.1. Let us denote by

$$
g_{\nu}(z)=\frac{1}{n} \sum_{k=1}^{n} \alpha_{k n} p_{\nu k}(z), \quad \alpha_{k n}=u_{k n}+i v_{k n},\left|\alpha_{k n}\right| \leq c_{1},
$$

where $p_{\nu k}(z)$ is defined by (2.13). Then

$$
\left\|g_{\nu}\right\|_{L_{p}} \leq c_{1} c_{2}, \quad 1<p<\infty
$$

where $c_{2}$ is an absolute constant depending only on $p$.

PROOF. Since $\left|\alpha_{k n}\right| \leq c_{1}$ it follows that

$$
\left|u_{k n}\right| \leq c_{1}, \quad\left|v_{k n}\right| \leq c_{1} .
$$

Also, it is known that

$$
t_{\nu, k}(\theta) \geq 0, \quad \frac{1}{n} \sum_{k=1}^{n} t_{\nu, k}(\theta)=1 .
$$

Thus it is easy to conclude that

$$
\left|\frac{1}{n} \sum_{k=1}^{n} u_{k n} t_{\nu k}(\theta)\right| \leq c_{1}, \quad\left|\frac{1}{n} \sum_{k=1}^{n} v_{k n} t_{\nu k}(\theta)\right| \leq c_{1} .
$$

Now by using a well-known theorem of Riesz [9, Theorem 2.4, p. 253], (3.5) and the formula

$$
\begin{aligned}
g_{\nu}(z)= & \frac{1}{n} \sum_{k=1}^{n} u_{k n} t_{\nu k}(\theta)-\frac{1}{n} \sum_{k=1}^{n} v_{k n} \tilde{t}_{\nu k}(\theta) \\
& +\frac{i}{n} \sum_{k=1}^{n} u_{k n} \tilde{t}_{\nu k}(\theta)+\frac{i}{n} \sum_{k=1}^{n} v_{k n} t_{\nu k}(\theta) .
\end{aligned}
$$

We immediately obtain (3.2). 
LEMMA 3.2. Let $f(z) \in C|| z \mid \leq 1]$. Then $I_{m}(f, z)$ as defined by (2.1), (2.3) satisfies the estimate

$$
\left\|I_{m}(f, z)\right\|_{L_{p}} \leq c_{p}\|f\|_{L_{\infty}}
$$

where $c_{p}$ depends only on $p$. Also, let

$$
Q_{n}(z)=\sum_{k=1}^{n} v_{k n m} B_{k, n, m}(z)
$$

where

$$
\left|v_{k n m}\right| \leq \varepsilon_{n m}
$$

Then we have

$$
\left\|Q_{n}(z)\right\|_{L_{p}} \leq \varepsilon_{n m} c_{4}(p) n^{-m} .
$$

Here $B_{k, n, m}(z)$ is defined by (2.4), (2.6).

From (2.15), (2.16), (2.3) and some simple calculations it follows that

$$
\begin{aligned}
& A_{k, n, m}(z)= \frac{z^{n}-1}{2 n} \lambda_{n-1, n, m}+l_{k, n}(z)\left(1-\left(z^{n}-1\right) \lambda_{n-1, n, m}\right) \\
&-\frac{z^{n}-1}{2 n}\left\{\sum_{j=1}^{n-1}\left(\lambda_{j-1, n, m}-2 \lambda_{j, n, m}+\lambda_{j+1, n, m}\right) j p_{j, k}(z)\right. \\
&\left.\quad+\left(\lambda_{n-1, n, m}-\lambda_{n, n, m}\right)(n-1) p_{n-1, k}(z)\right\}
\end{aligned}
$$

Also as a consequence of the $L_{p}$ convergence of the Lagrange interpolation process based on the $n$th roots of unity (Marcinkiewicz Theorem). Chapter 10 of [9], it follows that

$$
\left\|\sum_{k=1}^{n} f\left(z_{k n}\right) l_{k n}(z)\right\|_{L p} \leq c_{7}\|f\|_{L_{\infty}}, \quad 1<p<\infty .
$$

Now, on using (2.8), (2.9), (2.10), (3.11), (3.12), estimate (3.7) follows. Proof of (3.10) is on similar lines; there one uses (3.9), (3.8), (2.8), (2.9), (2.11), (3.12) and the following representation of the fundamental polynomials of the second kind. We omit the details.

$$
\begin{aligned}
B_{k, n, m}(z)=\frac{z^{n}-1}{2 n} z_{k n}^{m}\left[\begin{array}{l}
- \\
-
\end{array}\right. & \mu_{0, n, m}+\left(2 n l_{k n}(z)+1\right) \mu_{n-1, n, m} \\
& +(n-1) p_{n-1, k}(z)\left(\mu_{n-1, n, m}-\mu_{n, n, m}\right) \\
& \left.+\sum_{\nu=1}^{n-1} \nu\left(\mu_{\nu-1, n, m}-2 \mu_{\nu, n, m}+\mu_{\nu+1, n, m}\right) p_{\nu, k}(z)\right]
\end{aligned}
$$

In the proof of our theorems the next lemma [10] plays an important role. 
LEMMA 3.3. Let $f(z) \in C[|z| \leq 1]$. Let $w_{m}(f, \delta)$ be the generalized modulus of continuity of $f\left(e^{i \theta}\right), 0 \leq \theta \leq 2 \pi$. Then there exists a polynomial $F_{n}(z)$ of degree $\leq n-1$ such that for $|z| \leq 1$ we have

$$
\left|f(z)-F_{n}(z)\right| \leq c_{8} w_{m}(f, 1 / n)
$$

and

$$
\left|F_{n}^{(m)}(z)\right| \leq c_{9} n^{m} w_{m}(f, 1 / n) .
$$

4. Proof of Theorem 1. It is enough to prove Theorem 1 for $1<p$. (See $[9$, p. 25, Theorem 10.12].) Let $f(z) \in C[|z| \leq 1]$. We denote by $F_{n}(z)$ the polynomial of degree $\leq n-1$ satisfying (3.14) and (3.15). Then from (2.1)

$$
\begin{aligned}
f(z)-I_{n, m}(f, z)= & f(z)-F_{n}(z)+F_{n}(z)-I_{n, m}\left(F_{n}, z\right) \\
& +I_{n, m}\left(F_{n}-f, z\right)=f(z)-F_{n}(z)+I_{n, m}\left(F_{n}-f, z\right) \\
& +\sum_{k=1}^{n} F_{n}^{(m)}\left(z_{k n}\right) B_{k, n, m}(z),
\end{aligned}
$$

On using (3.14), (3.7) we have $(p>1)$

$$
\left\|I_{n, m}\left(F_{n}-f, z\right)\right\|_{L_{p}} \leq c_{p}\left\|F_{n}-f\right\|_{L_{\infty}} \leq c_{p} c_{8} w_{m}(f, 1 / n) .
$$

Also, from (3.15), (3.10), (3.9) we obtain

$$
\begin{aligned}
\left\|\sum_{k=1}^{n} F_{n}^{(m)}\left(z_{k n}\right) B_{k, n, m}(z)\right\|_{L_{p}} & \leq c_{9} n^{m} w_{m}(f, 1 / n) c_{4}(p) n^{-m} \\
& \leq c_{10} w_{m}(f, 1 / n) .
\end{aligned}
$$

Now, from (4.1), (4.2), (4.3) and (3.14) we have (1.3). This proves Theorem 1 for $1<p<\infty$.

The proof of Theorem 2 is on the same lines so we omit the details.

The author would like to express his deep appreciation to the referee for many helpful suggestions.

REMARKS. After the paper was sent for publication the author discovered that G. Francsics has also considered the problem of mean convergence of Birkhoff interpolation based on the roots of unity (to appear in J. Approximation Theory). He mentioned in this interesting paper our earlier results written jointly with $\mathrm{J}$. Szabados. In the present work the approach we have considered gives the solution to the problem of degree of convergence as well. (We also like to mention that for the purpose of simplicity we have limited ourselves to the most interesting case of Birkhoff interpolation $(o, m)$ only.)

\section{BIBLIOGRAPHY}

1. A. S. Cavaretta, A. Sharma and R. S. Varga, Hermite Birkhoff interpolation in the nth roots of unity, Trans. Amer. Math. Soc. 259 (1980), 621-628.

2. O. Kis, Notes on interpolation, Acta Math. Acad. Sci. Hungar. 11 (1960), 49-64.

3. G. G. Lorentz, K. Jetter and S. D. Riemenschneider, Birkhoff interpolation, Encyclopedia of Mathematics and its Application, Vol. 19, (see Chapter XII), 1983.

4. S. D. Riemenschneider and A. Sharma, Birkhoff interpolation at the $n$th roots of unity, Canad. J. Math. 33 (1981), 362-371. 
5. A. Sharma and P. Vertesi, Mean convergence of interpolation in roots of unity, SIAM J. Math. Anal. 14 (1983), 800-806.

6. J. Szabados, On some convergent interpolatory polynomials, Fourier Analysis and Approximation Theory (G. Alexits and P. Turan, eds.), vol. II, North-Holland, 1978, pp. 805-816.

7. J. Szabados and A. K. Varma, On an open problem of P. Turan concerning Birkhoff interpolation based on the roots of unity, J. Approximation Theory 47 (1986), 255-264.

8. P. Turán, On some open problems of approximation theory, J. Approximation Theory 29 (1980), 23-85.

9. A. Zygmund, Trigonometric series, vols. I, II, Cambridge Univ. Press, 1968.

10. A. Sharma and J. Szabados, Convergence rates for some lacunary interpolators on their roots of unity (preprint April 1987).

Department of Mathematics, University of Florida, Gainesville, Florida 32611 